\title{
Autoignition of Hydrogen-Air Mixture with Temperature and Composition Inhomogeneities
}

\author{
Gaurav Bansal* and Hong G. $\operatorname{Im}^{\dagger}$ \\ Department of Mechanical Engineering, University of Michigan, Ann Arbor, MI 48109-2125
}

\begin{abstract}
Autoigntion and front propagation behavior in Low Temperature Combustion (LTC) engine environments is investigated in this paper. First, non-reacting $3 \mathrm{D}$ engine simulations are conducted to investigate different mixture formation scenarios that might exist in LTC engines prior to autoignition. It is found that depending on the timing of fuel spray injection and the level of wall heat loss, two different scenarios might exist close to top-dead center (TDC): (1) early start of injection for which the intake stroke results in largely uncorrelated temperature $(T)$-equivalence ratio $(\phi)$ fields mostly due to wall heat loss; (2) late start of injection for which the compression stroke results in negatively correlated T- $\phi$ fields mostly due to evaporative cooling. Small-scale effects of these different mixture formation scenarios on the autoignition and subsequent front propagation are then studied using high-fidelity direct numerical simulation (DNS). For this purpose, high pressure hydrogenair mixture in constant volume with isotropic turbulence is investigated with detailed chemistry. Three cases with different initial T- $\phi$ fields are studied: case (A) baseline case with just temperature inhomogeneities and a uniform equivalence ratio field, case (B) uncorrelated T- $\phi$ fields and case (C) negatively-correlated T- $\phi$ fields. Numerical diagnostics are developed based on Damköhler number defined using the sensible enthalpy equation and an appropriately defined passive mixing time scale to decipher the different modes of heat release. It is found that majority of heat release in uncorrelated case and the baseline case occurs via premixed flame propagation, whereas the negatively correlated case ignites pretty much homogeneously.
\end{abstract}

\section{Nomenclature}

$u^{\prime} \quad$ Root mean square (RMS) velocity fluctuation

$L_{e, u} \quad$ Most energetic length scale of velocity fluctuation

$L_{11, u} \quad$ Integral length scale of velocity fluctuation

$T^{\prime} \quad$ Root mean square (RMS) temperature fluctuation

$L_{e, T} \quad$ Most energetic length scale of temperature fluctuation

$L_{11, T}$ Integral length scale of temperature fluctuation

$\phi^{\prime} \quad$ Root mean square (RMS) equivalence ratio fluctuation

$L_{e, \phi} \quad$ Most energetic length scale of equivalence ratio fluctuation

$L_{11, \phi}$ Integral length scale of equivalence ratio fluctuation

$D a_{\text {hsens }}$ Damköhler number based on sensible enthalpy equation

$D_{i} \quad$ Mass diffusivity of $i^{t h}$ species

$\lambda \quad$ Thermal conductivity of the mixture

$C_{p} \quad$ Mixture specific heat

$h_{o}^{i} \quad$ Standard specific enthalpy of formation of species $i$

$\omega_{i} \quad$ Reaction rate of species $i$

HRR Non-dimensional heat release rate

$t_{\text {mixH }}$ Average mixing time scale of specific total enthalpy

$t_{\text {mixz }}$ Average mixing time scale of mixture fraction

\footnotetext{
* Graduate Student, AIAA Student Member.

${ }^{\dagger}$ Associate Professor, AIAA Associate Fellow.
} 
$Z^{\prime} \quad$ Root mean square (RMS) mixture fraction fluctuation

$H^{\prime} \quad$ Root mean square (RMS) specific total enthalpy fluctuation

$\chi_{Z} \quad$ Mixture fraction dissipation rate

$\chi_{H} \quad$ Specific total enthalpy dissipation rate 


\section{Introduction}

Recent developments in internal combustion (IC) engine research have mainly focused on achieving low temperature combustion (LTC) in favor of superior efficiency and emission performance. ${ }^{1,2}$ A number of new engine concepts, such as homogeneous charge compression ignition (HCCI), controlled auto-ignition (CAI), and partially premixed compression ignition (PPCI), all fall into this new breed of IC engines design. While conventional HCCI refers to a type of combustion which typically has approximately homogeneous charge in terms of temperature and mixture composition, some degree of stratification is deliberately introduced ${ }^{3,4}$ in order to avoid extremely rapid pressure rise and heat release rates in the engine. Exhaust gas recirculation ${ }^{5}$ and multiple fuel injection ${ }^{6}$ are some of the techniques that are employed to introduce charge stratification in the engine cylinder. Moreover, some thermal stratification always exists in the cylinder due to wall heat loss. Due to the presence of turbulence, large scale stratification of charge leads to small scale inhomogeneities in both temperature and fuel mass fraction. Therefore, one of the major technical challenges in the development of LTC engines is the difficulties in controlling the start and subsequent phasing of combustion, because they essentially rely on the spontaneous autoignition of the mixture pockets that are subjected to varying degrees of flow/scalar inhomogeneities. At present, the combustion mode in LTC engines is not well understood, as both volumetric and front-like combustion modes can occur. ${ }^{7}$ In-depth understanding of the various modes of heat release which might occur in a stratified engine is therefore crucial for optimal design of these engines.

The objectives of the present study is to first identify possible mixture formation patterns close to topdead center (TDC) prior to autoignition in a typical LTC engine. To this end, non-reacting three-dimensional (3D) real engine simulations are performed using a multi-dimensional KIVA-3V code for internal combustion engine simulations. Depending on the fuel injection timing, exhaust gas recirculation, and the amount of wall heat loss, different correlations are found to exist between temperature and equivalence ratio at TDC. Essentially, it is found that two distinct scenarios exist: (1) early start of injection for which the intake stroke results in largely uncorrelated temperature and equivalence ratio fields mostly due to turbulence mixing and wall heat loss; (2) late start of injection for which the compression stroke results in negatively correlated temperature and equivalence ratio fields mostly due to evaporative cooling. Furthermore, insufficient mixing between hot residual gas which contain oxygen and the fresh charge can result in a negatively correlated temperature and equivalence ratio fields (see, for example, Fig. 3 of Ref. [5]).

The non-reacting engine simulations provide guidance to representative initial scalar fields to be studied for the autoignition characteristics. The ignition and front propagation events are simulated using direct numerical simulation (DNS) with detailed chemistry. Three different initial conditions are studied: Case (A) - a baseline case with only thermal inhomogeneities and a uniform equivalence ratio field. This configuration has been studied in a few recent studies; ${ }^{8-10}$ Case (B) - uncorrelated temperature and equivalence ratio fields; and Case $(\mathrm{C})$ - negatively correlated temperature and equivalence ratio fields. High pressure hydrogenair mixture is used and a constant volume configuration is adopted. Detailed hydrogen-air chemistry is employed. A two-dimensional homogeneous isotropic turbulence spectrum is imposed on the initial field. Main reason for using hydrogen-air mixture is to reduce the computational complexity which is already very high due to the need for resolving extremely thin fronts which occur at high pressure. However, hydrogen-air mixture is important in its own right for its practical relevance in blended hydrocarbon and other alternative fuels. Although turbulence is inherently three-dimensional, the present two-dimensional study is a first step in understanding the effects of inhomogeneities in both temperature and equivalence ratio which are representative of inhomogeneities in real LTC engines and provides valuable insights.

Due to the presence of both equivalence ratio and temperature inhomogeneities, various modes of heat release such as homogeneous autoignition, sequential autoignition (a spontaneous ignition front ${ }^{12}$ ), and premixed flame propagation are found to exist. Numerical diagnostics are developed to distinguish between these different modes of heat release. Passive diffusion outside of the reaction zone is characterized using a passive mixing time scale as is done in a previous study ${ }^{9}$ and active diffusion inside the front is characterized using a Damköhler number defined based on the relative magnitude of reaction and diffusion terms in the sensible enthalpy equation. A Damköhler number criterion is conditionally applied in regions of high heat release and gives the locations where the mode of heat release is diffusion dominant (i.e. a premixed flame propagation) and reaction dominant (i.e. a spontaneous ignition front or homogeneous autoignition). The paper is organized as follows. Next section presents the results of 3D non-reacting engine simulations. Section III describes the numerical implementation and initial conditions used for DNS. Section IV and V presents 1D and 2D DNS results, respectively. Finally, the Conclusions section summarizes the key findings of the study. 


\section{Non-Reacting Engine Simulations}

To understand mixture formation process in a realistic LTC engine, non-reacting simulations are conducted for a 3D 4-valve pentroof engine using KIVA-3V, which is a multi-dimensional reacting fluid dynamics solver used extensively for internal combustion engine simulations. ${ }^{5,13}$ The turbulence model is based on the Reynolds-Averaged-Navier-Stokes (RANS) approach, and the conventional $k-\epsilon$ model is adopted in this study. Table 1 lists the specifications and the operating conditions of the engine simulated. The mesh consists of about 50000 grid cells and is shown in Fig 1. Gasoline fuel is used to represent realistic spray evaporation behavior. Fuel is injected from the top of the cylinder head vertically downwards.

\begin{tabular}{|c|c|}
\hline Bore & $9.2 \mathrm{~cm}$ \\
\hline Stroke & $8.5 \mathrm{~cm}$ \\
\hline Compression ratio & 9.64 \\
\hline IVO & 28 BTDC \\
\hline IVC & 66 ABDC \\
\hline EVO & 112 ATDC \\
\hline EVC & 28 ATDC \\
\hline $\mathrm{T}_{\text {head }}$ & $400 \mathrm{~K}$ \\
\hline $\mathrm{T}_{\text {piston }}$ & $400 \mathrm{~K}$ \\
\hline $\mathrm{T}_{\text {cylinderwall }}$ & $400 \mathrm{~K}$ \\
\hline RPM & 1500 \\
\hline Intake temperature & $400 \mathrm{~K}$ \\
\hline
\end{tabular}

Table 1. Engine specifications and operating conditions.

Using the non-reacting simulations, the effects of fuel injection timing on mixture formation are studied. Of particular interest is the correlation between temperature and equivalence ratio fields close to TDC prior to autoignition. Towards this goal, two cases are studied with different injection timing. Case (kA) has a start of injection (SOI) timing of 270 degrees before TDC, and Case (kB) has SOI of 90 before TDC. Thus, Case $(\mathrm{kA})$ represents an early-injection scenario and Case $(\mathrm{kB})$ represents a late-injection scenario. In both cases, duration of injection was 48 crank angle degrees and the global equivalence ratio was fixed to 0.19. Both simulations were initiated at 375 degrees before TDC (BTDC) and the initial turbulence kinetic energy was $10 \%$ of kinetic energy based on mean piston speed.

The results for Case (kA), the early injection case, are first examined. Figure 2 shows the fuel mass fraction and temperature fields at 15 degrees BTDC, in a plane normal to the cylinder axis close to the top wall of the cylinder. It is observed that temperature is high in the middle and low close to the walls due to wall heat loss. It can be seen that apart from the slight negative correlation on the right boundary due to the evaporation cooling effect, the two fields are largely uncorrelated with each other. This is owing to the turbulence mixing and wall heat loss effects. This is further demonstrated in Fig. 3 which shows the scatter plot of fuel mass fraction and temperature also at 15 degrees BTDC. The scatter plot includes all the data points inside the engine cylinder and shows that the fields are mostly uncorrelated. For most of the cells in the cylinder, the temperature varies between about $700 \mathrm{~K}$ and $800 \mathrm{~K}$, and the fuel mass fraction varies between 0 to about 0.03 . The mass-based stoichiometric fuel to air ratio for gasoline is 0.0672 , implying that the equivalence ratio in the cylinder varies between 0 to 0.46 .

Next, the results for Case $(\mathrm{kB})$, the late-injection case, are examined. Figure 4 shows the fuel mass fraction and temperature fields at 15 degrees BTDC, in the same cross-sectional plane of the cylinder as the previous case. Unlike Case $(\mathrm{kA})$, the temperature and fuel mass fraction fields are negatively-correlated to a large extent. At a location slightly to the right of center, the fuel has evaporated and the temperature has reduced due to evaporative cooling effect. In the periphery of evaporated fuel region, temperature is high and fuel mass fraction is almost zero. Wall heat loss effects are again seen in the thin region near the wall where temperature is lower. Figure 5 shows the scatter plot of fuel mass fraction and temperature fields at 15 BTDC for Case $(\mathrm{kB})$. All the cells within the cylinder are represented in this scatter plot. It is evident that there exists a strong negative correlation between the two fields. The lower constant temperature branch of the scatter plot represents the thermal boundary layer near the walls where the temperature is lower due 


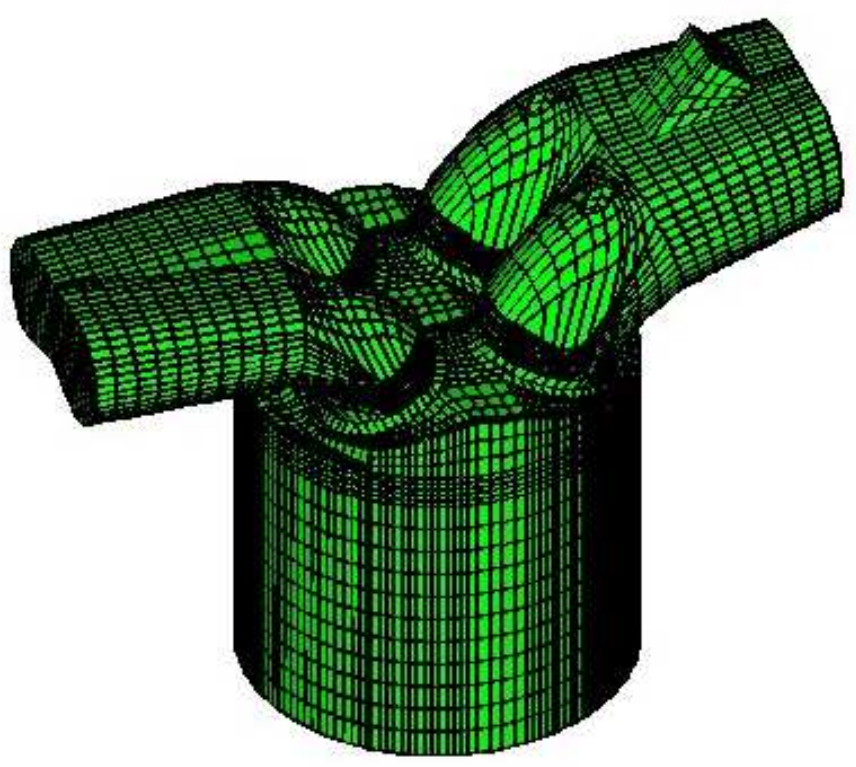

Figure 1. Pentroof engine mesh with about 50000 cells adopted for KIVA-3V simulations.
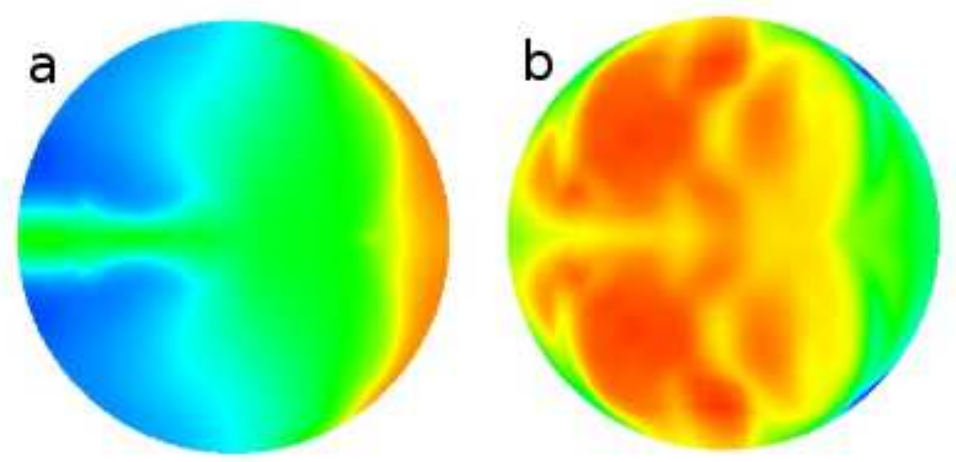

Figure 2. (a) Fuel mass fraction, levels ranging from 0 (blue) to 0.03 (red), (b) temperature, levels ranging from $680 \mathrm{~K}$ (blue) to $820 \mathrm{~K}$ (red) for Case (kA). Shown in a plane normal to cylinder axis close to top wall of cylinder, 15 degrees BTDC. 


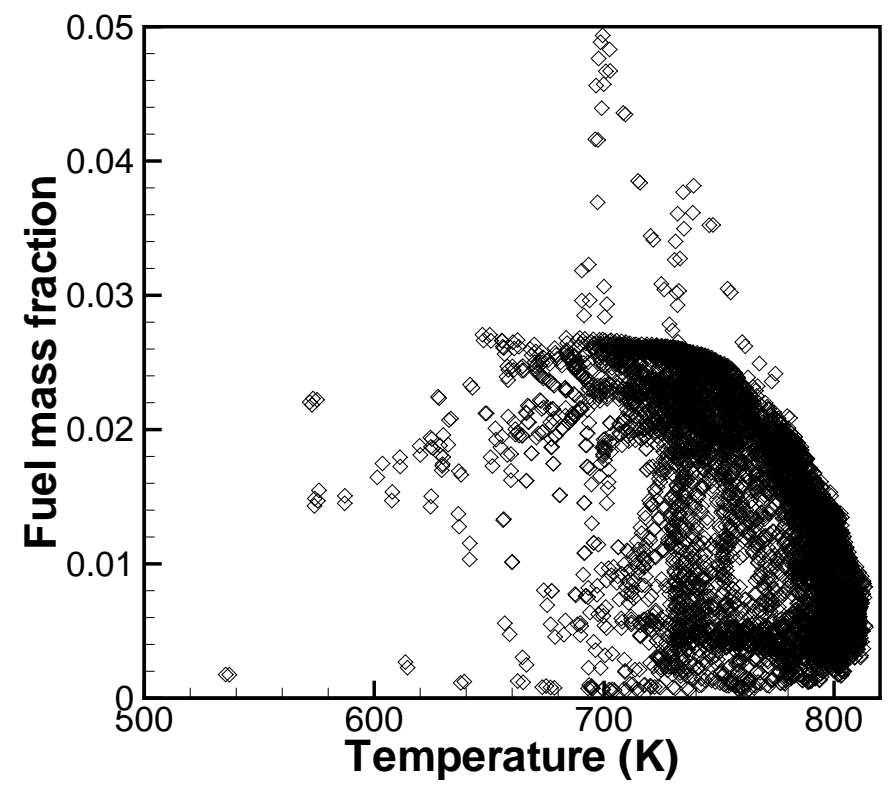

Figure 3. Scatter plot of temperature against fuel mass fraction at 15 degrees BTDC for Case (kA).

to heat loss. In this case, since the fuel is injected very late, there is still a large variation in fuel mass fraction field at 15 degrees BTDC, with the equivalence ratio varying from 0 to 1.47 inside the cylinder. Note that about $60 \%$ of the fuel is evaporated at 15 BTDC. Temperature again varies from about 700-800 $\mathrm{K}$ for majority of cells within the cylinder. Due to late fuel injection there is not enough time for turbulence mixing to modulate the effects of evaporative cooling and this causes the temperature and equivalence ratio fields to be negatively correlated.
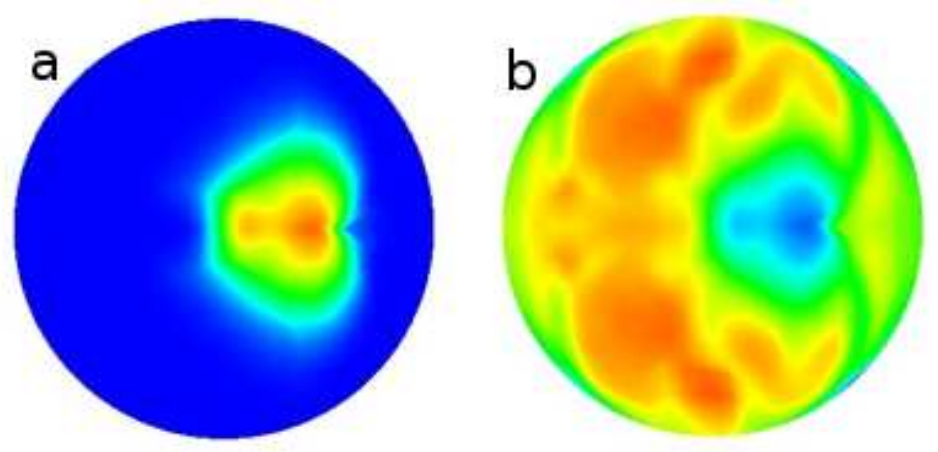

Figure 4. (a) Fuel mass fraction, levels ranging from 0 (blue) to 0.08 (red), (b) temperature, levels ranging from $700 \mathrm{~K}$ (blue) to $840 \mathrm{~K}$ (red) for Case (kB). Shown in a plane normal to cylinder axis close to top wall of cylinder, 15 degrees BTDC.

Apart from these two cases, exhaust gas recirculation (EGR) can also result in a negatively correlated equivalence ratio and temperature fields for an early-injection or port-fuel injection case. If the overall mixture is lean, the hot EGR stream contains large levels of oxygen. Incomplete mixing between hot EGR stream and fresh charge then results in hot pockets with excess oxygen and cold pockets with lower levels of oxygen. This creates a negatively correlated temperature-equivalence ratio field. An illustration of this is shown in Babajimopolous et al. ${ }^{5}$ (See Fig. 3 of Ref. [5]).

Having understood the different mixture formation scenarios that might exist in a real engine prior to autoignition, we next study the small-scale implications of these on autoignition and subsequent front propagation using high-fidelity DNS. There remains a question as to whether the large scale correlations observed 


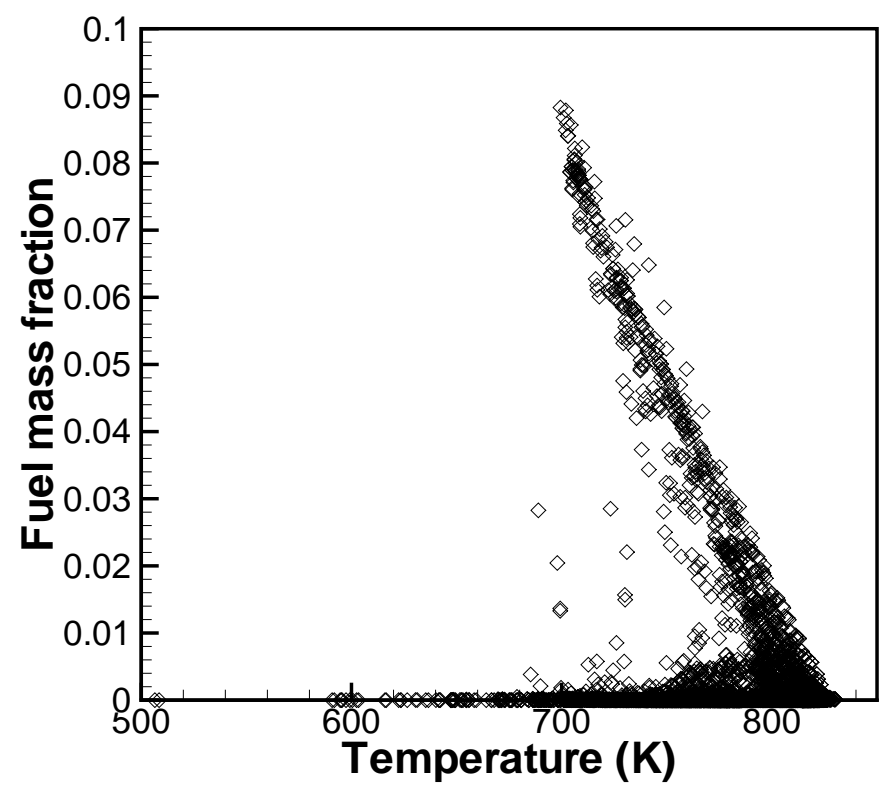

Figure 5. Scatter plot of temperature against fuel mass fraction at 15 degrees BTDC for Case (kB).

in the RANS simulations will result in the same degree of small subgrid-scale correlations. Considering turbulent mixing characteristics, it is reasonable to assume that uncorrelated large scales will result in uncorrelated small scales. On the other hand, even if large scales are correlated, small scales might still be uncorrelated to some extent due to turbulence mixing at small scales. Further investigation is needed to provide insights into this issue.

\section{Numerical Implementation and Initial Conditions for DNS}

In this section, we present the solution algorithm and initial conditions for DNS. The full compressible Navier-Stokes, species, and energy equations for a reacting gas mixture are solved using a fourth-order Runge-Kutta method for time integration and an eight-order explicit spatial difference scheme. ${ }^{14,15}$ For DNS runs, hydrogen is used as a fuel and detailed hydrogen-air chemical mechanism with 9 species and 22 chemical reactions developed by Mueller et al. ${ }^{11}$ is used. Thermal conductivity, $\lambda$, is given as a function of temperature following Smooke and Giovangigli. ${ }^{16}$ The mixture specific heat $C_{p}$ is a function of local mixture composition, $C_{p}=\sum_{k} C_{p, k} Y_{k}$, where each $C_{p, k}$ is curve-fitted as a function of temperature using the Chemkin thermodynamic database. ${ }^{17}$ The molecular viscosity is temperature dependent and constant Lewis numbers for individual species are used.

For both 1D and 2D simulations, periodic boundary conditions are employed at all the boundaries to represent the constant volume ignition process. The computational domain for 1D simulations consists of 400 grid points in a $1 \mathrm{~mm}$ domain, which gives a spatial resolution of $2.5 \mu \mathrm{m}$. The computational domain for 2D simulations is a $4.1 \mathrm{~mm} \times 4.1 \mathrm{~mm}$ square with $960 \times 960$ grid points, which gives the grid resolution of $4.3 \mu \mathrm{m}$. Such a fine resolution is required for resolving the thin propagating fronts which result due to the high pressure conditions employed in this study. The $2 \mathrm{D}$ grid used is similar to the one used in previous studies. ${ }^{8,9}$

The mean flow velocities for all cases are set to $0 \mathrm{~cm} / \mathrm{s}$ initially. The following description applies to the $2 \mathrm{D}$ simulations. At initial time, turbulence velocity fluctuations are superimposed on the stationary mean velocity field based on the Passot-Pouquet turbulent kinetic energy spectrum function: ${ }^{18}$

$$
E(k)=\frac{32}{3} \sqrt{\frac{2}{\pi}} \frac{u^{\prime 2}}{k_{e}}\left(\frac{k}{k_{e}}\right)^{4} \exp \left[-2\left(\frac{k}{k_{e}}\right)^{2}\right]
$$


where $k$ is the wave number magnitude, $k_{e}$ is the most energetic wave number and $u^{\prime}$ is the rms velocity fluctuation. In all the cases, the mean initial temperature is $1070 \mathrm{~K}$, mean hydrogen-air equivalence ratio is 0.1 and the uniform initial pressure is $41 \mathrm{~atm}$. Random temperature and composition fields are superimposed on the mean temperature and composition fields respectively. The temperature and equivalence ratio spectrums similar to turbulence kinetic energy spectrum are used to specify the characteristic scales of initial hot spots and initial rich fuel pockets respectively.

The initial autocorrelation integral length scale of the velocity fluctuations $\left(L_{11, u}\right)$ is $0.34 \mathrm{~mm}$ and the most energetic length scale $\left(L_{e, u}\right)$ is $1.0 \mathrm{~mm}$. The velocity RMS $\left(u^{\prime}\right)$ is $0.5 \mathrm{~m} / \mathrm{s}$. This gives integral time scale $\left(t_{11}=L_{11, \mathrm{u}} / u^{\prime}\right)$ of turbulent fluctuations as about $0.7 \mathrm{~ms}$, and the integral scale Reynolds number of 51. In a typical engine, $u^{\prime}$ values are found to be of the order of $5 \mathrm{~m} / \mathrm{s}$, and $L_{11, \mathrm{u}}$ is of the order of $6 \mathrm{~mm} .{ }^{19}$ This gives the turbulent integral time scale as $1.2 \mathrm{~ms}$ and the integral scale Reynolds number as 9000 for a typical engine. Thus the integral time scale employed in DNS is comparable to that of a real engine, while the integral scale Reynolds number is about 180 times smaller than that in an engine. The homogeneous ignition delay time for hydrogen ignition computed at mean temperature of $1070 \mathrm{~K}$ and mean equivalence ratio of 0.1 is found to be $2.46 \mathrm{~ms}$, which is also comparable to ignition delay times in a typical engine. In our earlier studies of autoignition with velocity fluctuations, ${ }^{20,21}$ we found that the turbulence eddy which has a turnover time comparable to ignition delay has maximum interaction with the ignition chemistry. Since the turbulence integral time scale employed in DNS is comparable to ignition delay, the integral scale eddies are the ones that primarily interact with ignition chemistry.

The temperature and equivalence ratio fields have an RMS fluctuation of $15 \mathrm{~K}$ and 0.05 , respectively. The autocorrelation integral length scale and most energetic length scale are $0.45 \mathrm{~mm}$ and $1.32 \mathrm{~mm}$, respectively, for both temperature and equivalence ratio fields. Note that, as specified, $T^{\prime} / \bar{T}(=0.014)$ is much smaller than $\phi^{\prime} / \bar{\phi}(=0.5)$. This way ignition delay variation due to temperature fluctuation alone is comparable to that due to equivalence ratio fluctuation alone, considering the much stronger exponential dependence of ignition delay on temperature.

The length scales of hot spots ${ }^{7}$ and rich fuel pockets observed in an engine are approximately five times larger than those specified in the present DNS study. However, the key turbulence mixing time scale, i.e. the integral time scale, is matched with that in a typical engine. Furthermore, the level of variation in temperature (about $100 \mathrm{~K}$ ) considered in DNS is comparable with that obtained in engine simulation results in the previous section, implying that $T^{\prime}$ is comparable between DNS and a typical engine. Therefore, since the key mixing time scale and $T^{\prime}$ are matched between DNS and a typical engine, local temperature gradients will also be comparable between DNS and a typical engine (see the definition of mixing time scale in Eq. (6)). In a previous study, ${ }^{8}$ local temperature gradient is shown to be the key parameter affecting molecular transport in high heat release regions. Thus, as demonstrated by Hawkes et al. ${ }^{10}$ a change in initial length scales may not affect the heat release behavior if the turbulence mixing time scale and $T^{\prime}$ are kept fixed. Based on this reasoning, the heat release behavior observed in the present DNS study is expected to be relevant in a real engine. The larger Reynolds number present in the engine will only contribute towards producing a larger range of scales.

Based on the insights gained from non-reacting engine simulations in Section II, three cases with different initial conditions are studied for 2D DNS studies. Case $(2 \mathrm{~A})$ is the baseline case with only temperature inhomogeneities and a uniform equivalence ratio of 0.1 ; Case $(2 \mathrm{~B})$ is with uncorrelated temperature and equivalence ratio fields; and Case (2C) is with negatively correlated temperature and equivalence ratio fields. Figure 6 shows the initial temperature field which is same for all three cases. The figure shows how the hot and cold spots of different length scales are superimposed on the mean temperature field. Figure 7 shows the scatter plot of initial equivalence ratio and temperature fields for the three cases. Note that in Cases (2B) and $(2 \mathrm{C})$ the equivalence ratio distribution has to be truncated at the lean end to prevent it from becoming negative. The variation in equivalence ratio specified in Cases $(2 \mathrm{~B})$ and $(2 \mathrm{C})$ is smaller than that observed in non-reacting engine simulations in the last section. If equivalence ratios greater than one are present, which is more probable for the late-injection case, there is a possibility of formation of diffusion flames in the engine which is not captured by DNS. High equivalence ratios are not employed in DNS because in high pressure hydrogen-air mixture there is a tendency to form shock waves at high equivalence ratios which the present DNS grid cannot resolve. 


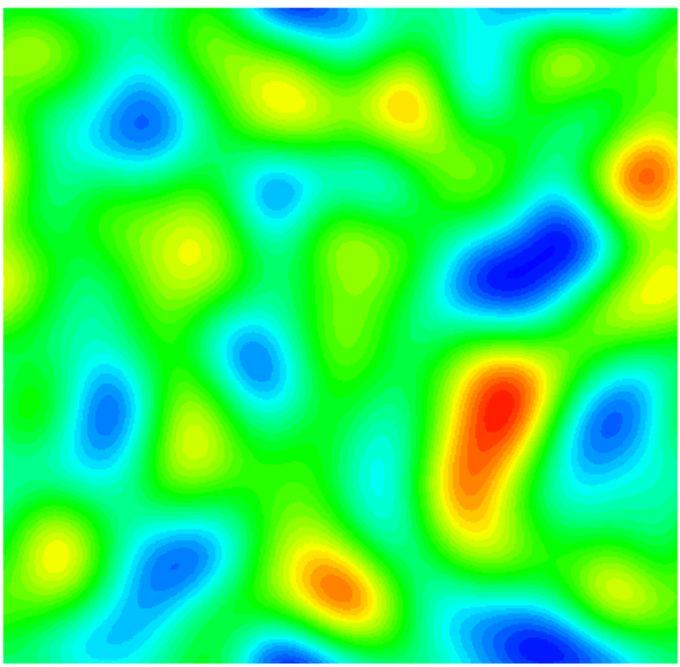

Figure 6. Initial temperature field for Cases (2A), (2B), and (2C), levels ranging from $1033 \mathrm{~K}$ (blue) to $1116 \mathrm{~K}$ (red).
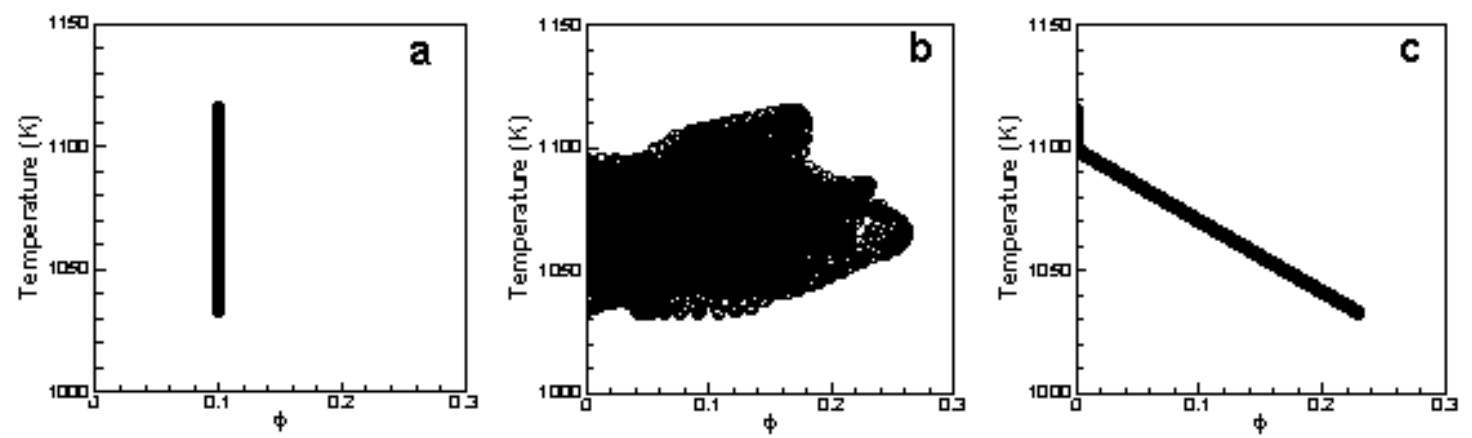

Figure 7. Temperature-equivalence ratio scatter plot at initial time for a: Case (2A), b: Case (2B), and c: Case (2C). 


\section{One-Dimensional Simulation Results}

As a first step towards understanding the complex autoignition process in inhomogeneous mixtures, one-dimensional calculations are conducted. Ignition and front-propagation regimes in non-homogeneous one-dimensional configuration has been investigated in some previous studies..$^{22-24}$ The 1D results will help in understanding more complex two-dimensional results which will be shown in the next section. Three cases with different initializations are studied as shown in Fig. 8. For all cases, mean temperature and pressure are fixed to $1095 \mathrm{~K}$ and $41 \mathrm{~atm}$, respectively, and a sinusoidal temperature field is imposed on the mean temperature field. Case (1A) has an amplitude of temperature fluctuation of $10 \mathrm{~K}$ and Case (1B) has that of $100 \mathrm{~K}$. Both Cases (1A) and (1B) have a uniform equivalence ratio of 0.1. Case (1A) is similar to one of the hot pockets of the 2D baseline case (Case (2A)) described in Section III. Case (1C) has amplitude of temperature fluctuation of $25 \mathrm{~K}$ and an sinusoidal equivalence ratio fluctuation of amplitude 0.05 is also imposed on top of mean equivalence ratio field of 0.05 . The equivalence ratio fluctuation is out of phase of the temperature fluctuation such that there is a negative correlation between them. Case (1C) represents one of the hot-lean pockets of the negatively correlated 2D case (Case $(2 \mathrm{C})$ ) defined in Section III.

Figure 9 shows the time sequence of non-dimensional heat release rate (HRR) profiles for the three cases. For Cases (1A) and (1B), the ignition kernel is initiated in the hot center region from which two reaction fronts emanate and propagate onto both sides. In Case (1C), the combustion event occurs in a totally different manner. Because of the lack of fuel in the center of domain, ignition does not initiate there although the temperature is maximum in center. After a prolonged ignition delay, longer than that of Cases (1A) and (1B), ignition starts and HRR increases from the sides, and subsequently fuel and radicals diffuse to the center, causing the center region to ignite. Due to the higher temperature, the center region catches up rapidly with the peripheral region such that the entire domain ignites in a roughly homogeneous manner. The solid lines in Fig. 9 in Case (1C) represents the rising portion of heat release rate and dashed lines indicate the times after the peak heat release rate due to the consumption of fuel in the domain. There is no clear evidence of front propagation in Case (1C).
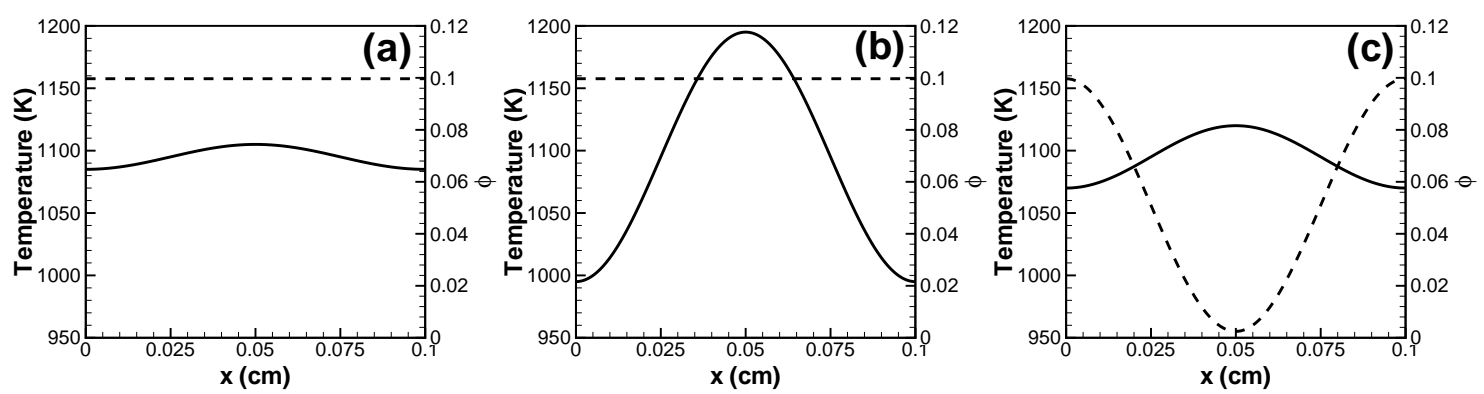

Figure 8. Initial temperature and equivalence ratio profiles for (a) Case (1A), (b) Case (1B), and (c) Case (1C)

Figure 10 shows the plot of spatially integrated non-dimensional heat release rate normalized with the total heat release for each case. Since total heat release is different for the three cases, normalization is done with total heat release for each case so that the area under each of the curves in Fig. 10 is unity. Distinct temporal behavior is observed for integrated HRR for the three cases. Case (1B) which has the highest maximum temperature ignites first, followed by Case (1A) and finally Case (1C). For Case (1B), heat release is very sharp and abrupt in the beginning and has a very long tail at the end. On the other hand, for Case $(1 \mathrm{~A})$, heat release rate slowly rises initially but the end charge is consumed quite rapidly. This suggests that the reaction front which consumes the end charge propagates at a much slower speed in Case (1B) compared to Case (1A). The initial rise of heat release rate is sharper in Case (1B) due to the presence of higher temperature in the center of the domain. Peak heat release rate is higher for Case $(1 \mathrm{~A})$ than for Case (1B). Case (1C) shows entirely different behavior, in that the ignition delay is much longer and the peak heat release rate is much lower. This is because the mean equivalence ratio is lower in Case $(1 \mathrm{C})$ compared to Cases (1A) and (1B) and also due to the fact that high-temperature regions have very low equivalence ratio to begin with for this case.

To identify the nature of front propagation, a computational diagnosis is now conducted. In a recent 

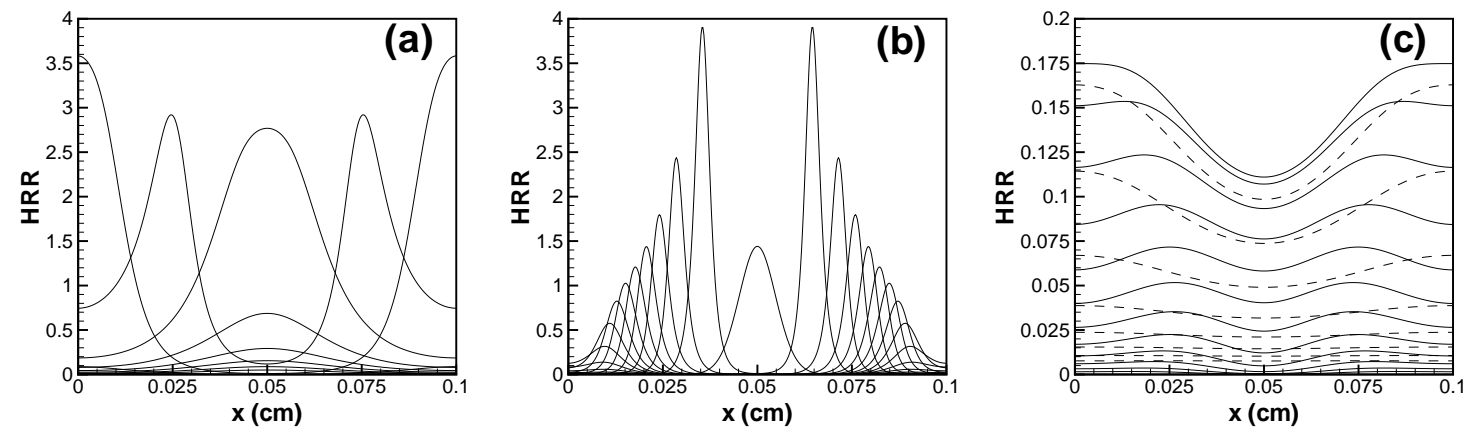

Figure 9. HRR profiles at various time instants during the entire combustion event for (a) Case (1A), (b) Case (1B), and (c) Case (1C). The ignition front propagates inside out for Cases (1A) and (1B), while HRR increases throughout the entire region in Case (1C).

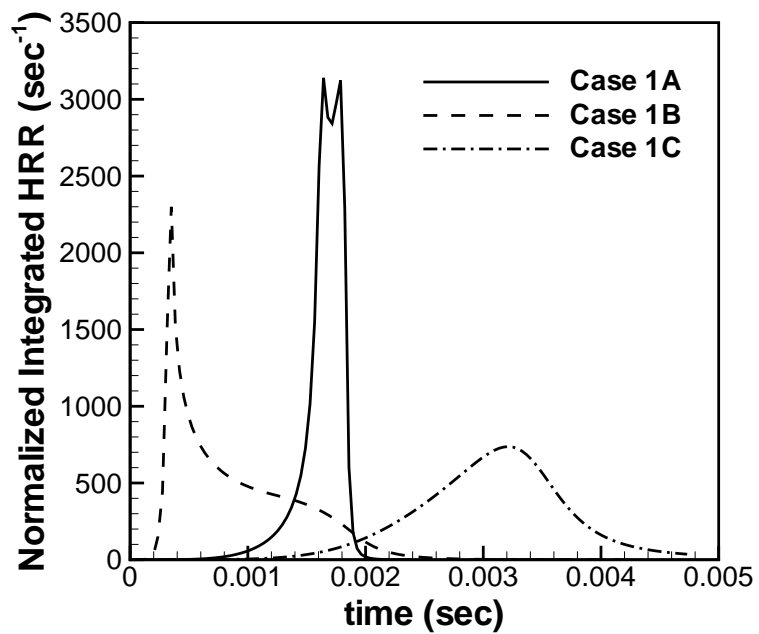

Figure 10. Normalized integrated HRR as a function of time cases (1A), (1B) and (1C). 
previous study by Chen et al. ${ }^{9}$ the ratio of local instantaneous Zel'odovich front speed ${ }^{12}$ to the laminar deflagration speed was used to identify the relative dominance between the deflagration and spontaneous ignition front propagation. However, using an unstretched steady flame speed in such a transient event is questionable. Furthermore, the laminar flame speed is not constant in the present study in the presence of mixture composition variations. Therefore, an alternative criterion is proposed. Considering that all the effects of diffusion and reaction are inherent in the conservation equations of energy and species, we define a Damköhler number based on the sensible enthalpy equation as follows:

$$
\mathrm{Da}_{\mathrm{hsens}}=\left|\frac{\sum_{i} h_{o}^{i} \omega_{i}}{\nabla \cdot\left(-\lambda \nabla T+\sum_{i} h_{s}^{i} \overline{J_{i}}\right)}\right|
$$

where the diffusive flux of species- $i, \overline{J_{i}}$, is given as:

$$
\overline{J_{i}}=-\rho D_{i} \nabla Y_{i}
$$

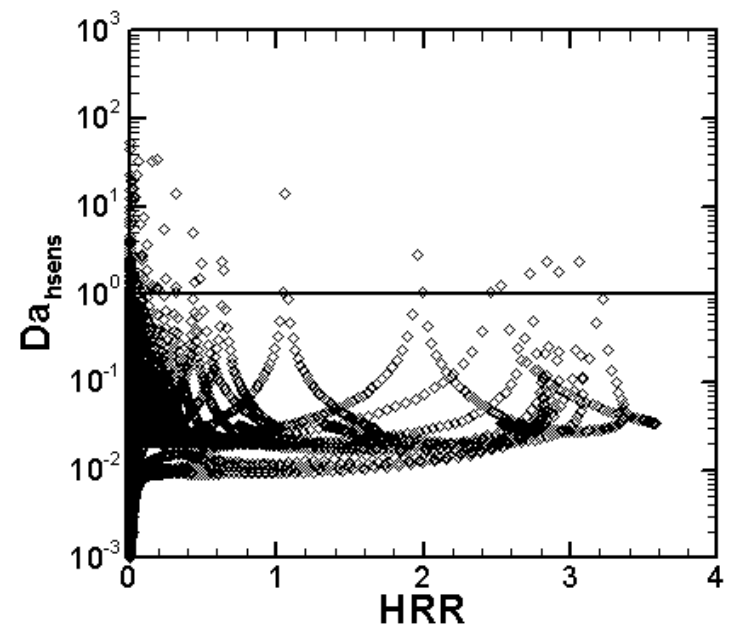

Figure 11. Scatter plot of Dahsens against HRR for Case (1A). Data points correspond to all spatial locations at various time instants during the combustion event.

The definition given in Eq. 2 includes the effect of both heat conduction and differential diffusion. A value of Dahsens less than one indicates a stronger prevalence of diffusion compared to reaction. Note that this definition is meaningful only when applied to regions of high heat release rate. In the regions of almost no heat release, the small Dahsens value simply represents a trivial nonreactive condition. Figure 11 shows the scatter plot of local non-dimensional heat release rate (HRR) against local $\mathrm{Da}_{\mathrm{hsens}}$, for various times during the ignition event, for Cases (1A). The Dahsens $=1$ is also drawn. As explained above, the data points whose HRR value is less than $10 \%$ of the maximum HRR (approximately HRR $<0.36$ for this case) are considered nonreactive and thus are not of interest. For HRR greater than 0.36 , it is found that a significant number of data points have a Dahsens $>1$, implying that the reaction front is characterized as a spontaneous ignition front as opposed to a premixed deflagration.

Figures 12 and 13 show the similar scatter plot for Cases (1B) and (1C). For Case (1B), almost all data points have Dahsens $<1$. Thus, diffusion is dominant compared to reaction in the reaction fronts. This indicates that the ignition event is characterized by a premixed flame propagation. On the other hand, Case (1C) shows a greater number of data points with Dahsens much greater than one. This case is almost completely dominant by reaction and represents a case of homogeneous autoignition. The Damköhler number criterion will be employed in the $2 \mathrm{D}$ simulations in the next section. 


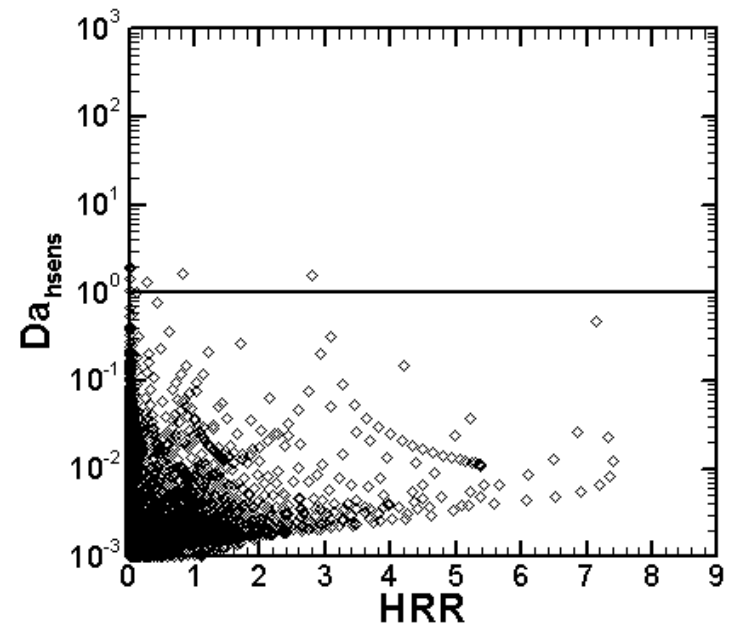

Figure 12. Scatter plot of Dahsens against HRR for Case (1B). Data points correspond to all spatial locations at various time instants during the combustion event.

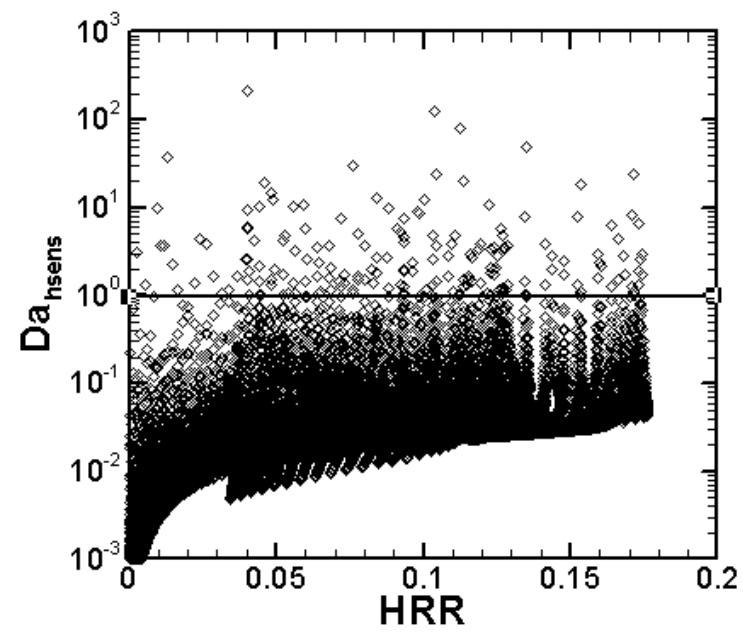

Figure 13. Scatter plot of $\mathrm{Da}_{\mathrm{hsens}}$ against HRR for Case (1C). Data points correspond to all spatial locations at various time instants during the combustion event. 


\section{Two-Dimensional Simulation Results}

In this section, we present the results of two-dimensional simulations. As mentioned in section III, three different cases are studied here. Case $(2 \mathrm{~A})$ is the baseline case with just temperature inhomogeneities and a uniform equivalence ratio of 0.1 . Case $(2 \mathrm{~B})$ is the case with initially uncorrelated temperature and equivalence ratio fields. This case represents the early-injection scenario shown in section II. Finally, Case $(2 \mathrm{C})$ is the case with initially negatively correlated temperature and equivalence ratio fields, representing the late-injection scenario of section II. Homogeneous isotropic turbulence is superimposed on the stationary mean velocity field at initial time.

Figure 14 shows the non-dimensional heat release rate (HRR) contours for the three cases $(2 \mathrm{~A}),(2 \mathrm{~B})$ and $(2 \mathrm{C})$ at three different time instants corresponding to $10 \%, 50 \%$ and $90 \%$ of total heat release. All the plots shown in this figure have same color scheme and the HRR levels vary from 0 (blue) to 9 (red). The top row shows the contours for Case $(2 \mathrm{~A})$. Thin reaction fronts are observed at $10 \%$ heat release, and a weak ignition kernel is seen at top left corner of the domain. The ignition front is initiated from an ignition kernel at the maximum temperature locations, and expands and consumes neighboring charge. The constant volume simulation causes the pressure to rise as the front expands. At $50 \%$ heat release point, a large number of reaction zones are developed. Still at this point, most of the heat release occurs in thin front like regions. Finally, at $90 \%$ heat release, much thicker reaction zones are seen, at which time the end charge is consumed almost homogeneously.

The middle row of Fig. 14 presents the results for Case (2B). The development of ignition kernel and subsequent front propagation is similar to Case (2A). Case (2B), however, shows a much more connected reaction front at $10 \%$ heat release point. The subsequent image at $50 \%$ heat release is also very similar to Case (2A) in that the heat release occurs mainly in thin regions. At $90 \%$ heat release point, however, heat release is more widespread in both thin and thick regions. This suggest that Case (2B) exhibits a mixed mode ignition with both front-like propagation and homogeneous combustion simultaneously present in the domain. This is in contrast to Case $(2 \mathrm{~A})$ where the bulk of the end charge was consumed in a homogeneous manner.

Finally, the bottom row shows the results for Case (2C), which shows a drastically different ignition event. At $10 \%$ heat release, no front-like propagation is seen, and heat release occurs homogeneously. At $50 \%$ heat release, thick structures are seen, implying that the heat release occurs almost homogeneously. Such a homogeneous evolution of heat release is sustained throughout until $90 \%$ heat release. Therefore, it is evident that Case $(2 \mathrm{C})$ represents a typical homogeneous ignition mode without any front propagation. This is because the hot temperature regions have very low fuel available due to the negative correlation. Therefore, the radical build-up slowly starts outside of the hot temperature zones in the bulk of the mixture, and eventually the diffusion of fuel and radicals leads the hotter temperature zones to ignite subsequently.

Figure 15 shows the normalized integrated HRR as a function of time for the three cases. As in 1D results, the normalization is done with the total heat release for each case so that the area under each of these curves is unity. Compared to Case (2A), the ignition delay is advanced for Case (2B) and delayed for Case $(2 \mathrm{C})$. In Case (2B), since the temperature and equivalence ratio are uncorrelated, there are regions in the domain which have high temperature as well as high equivalence ratio. This causes the ignition in these regions to initiate very early. On the other hand, in Case (2C) there is a prolonged ignition delay since ignition is initiated outside of the local hot spots. The duration of heat release is also increased for Case $(2 \mathrm{~B})$ and decreased for Case (2C) compared to Case (2A). Consequently, peak heat release rate is highest for Case (2C) and lowest for Case (2B).

The various time scales involved during the ignition event are investigated in detail. First, similar to Ref. [9], we define average passive mixing time scales of mixture fraction $(Z)$ and specific total enthalpy $(H)$ scalars:

$$
\begin{aligned}
\mathrm{t}_{\text {mixZ }} & =\frac{Z^{\prime 2}}{\widetilde{\chi_{Z}}} \\
\mathrm{t}_{\text {mixH }} & =\frac{H^{\prime 2}}{\widetilde{\chi_{H}}}
\end{aligned}
$$

Here mixture fraction is defined according to Bilger's definition. ${ }^{25} Z^{\prime}$ and $H^{\prime}$ are the RMS fluctuation of mixture fraction and enthalpy, respectively. Symbol $\sim$ denotes volume averaging. $\chi_{Z}$ and $\chi_{H}$ are the scalar 

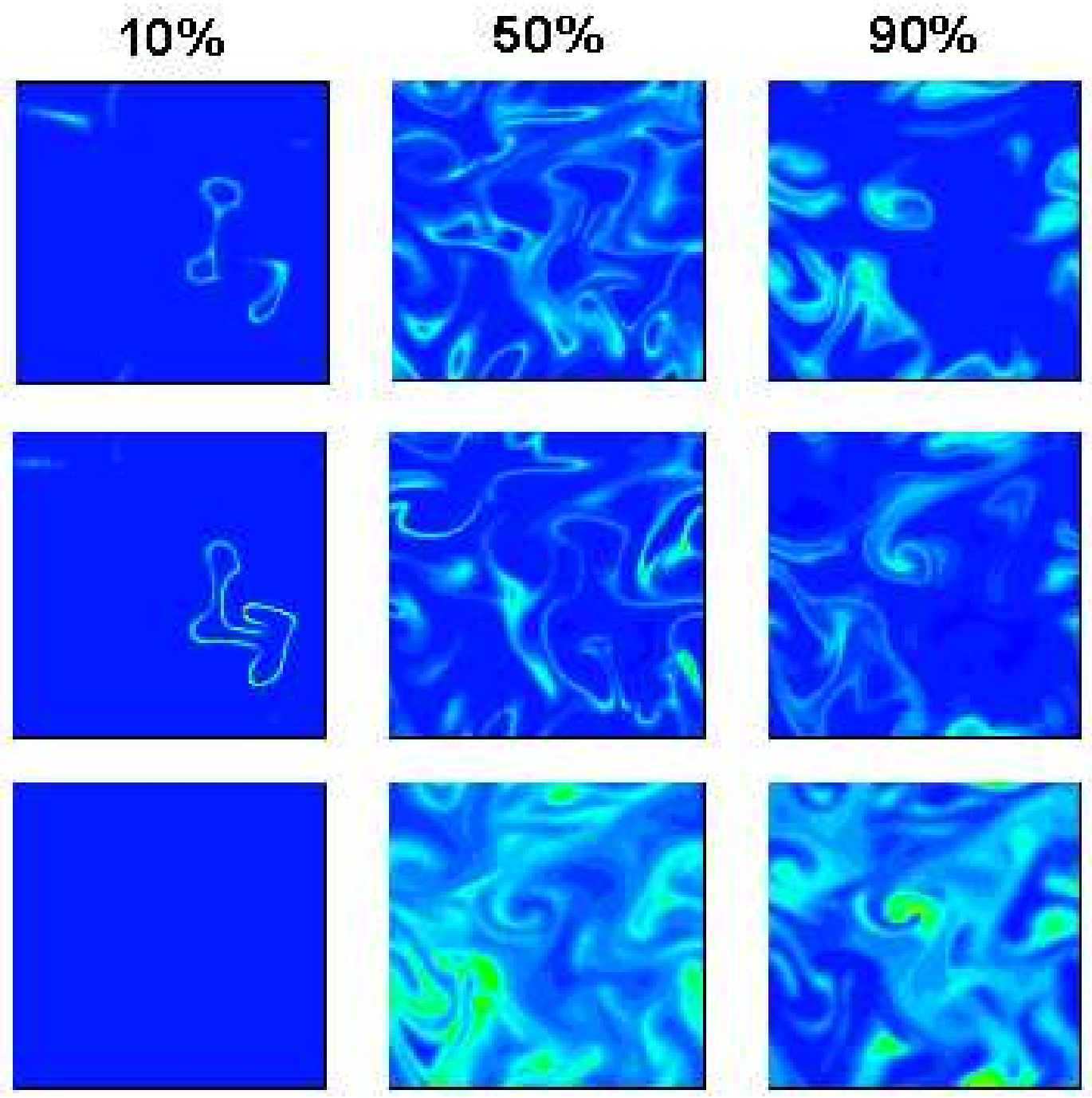

Figure 14. HRR contours at 10\%, $50 \%$ and $90 \%$ heat release points. Top row: Case (2A), middle row: Case (2B), bottom row: Case $(2 \mathrm{C})$. Levels ranging from 0 (blue) to 9 (red). 


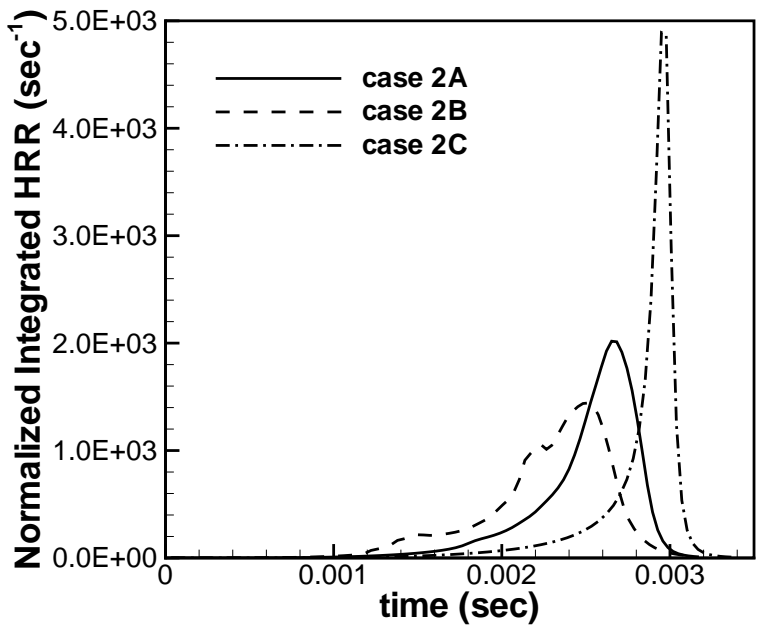

Figure 15. Normalized integrated HRR as a function of time for Cases (2A), (2B) and (2C).

dissipation rates based on mixture fraction and enthalpy scalars, respectively, defined as:

$$
\begin{gathered}
\chi_{Z}=2 \alpha|\nabla Z|^{2} \\
\chi_{H}=2 \alpha|\nabla H|^{2}
\end{gathered}
$$

The passive mixing time scale essentially gives a measure of how fast the passive scalars are mixing. A comparison of passive mixing time scale with the ignition delay shows whether the scalars will mix significantly in the duration of ignition delay. Unlike the Damköhler number criterion presented Section IV, this passive mixing time scale is applied to the entire computational domain.

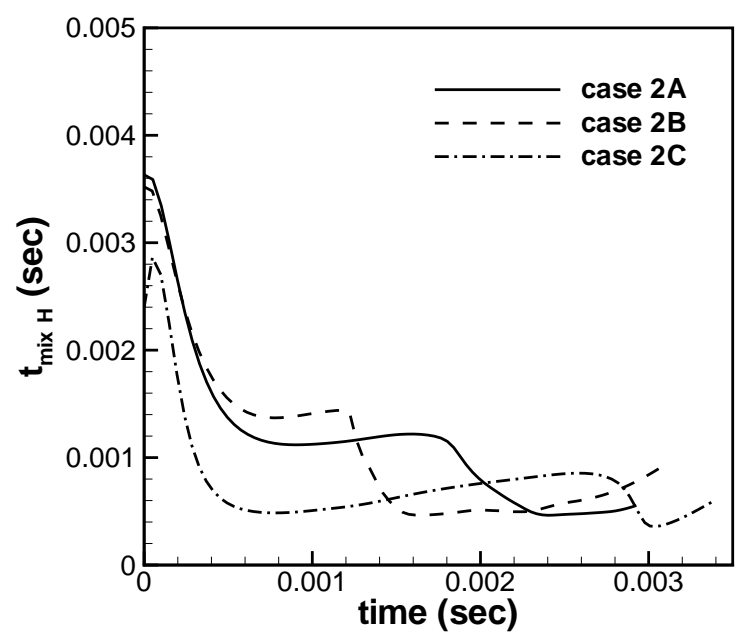

Figure 16. Average mixing time scale of specific total enthalpy as a function of time for cases (2A), (2B) and (2C).

Figure 16 shows the passive mixing time scale of $H$ scalar for the three cases. The initial mixing time scale of $H$ is almost identical for Cases $(2 \mathrm{~A})$ and $(2 \mathrm{~B})$, while Case $(2 \mathrm{C})$ has a lower initial value of $t_{\text {mixH }}$. This is because, although the initial temperature distribution is the same for the three cases, the initial fuel mass fraction profiles are different, causing the differences in the initial enthalpy profiles. Soon after the start of simulation, turbulent straining increases the gradients and reduces $t_{m i x H}$ for all the three cases. This behavior was also observed in Ref. [9]. Afterwards, there is a slight rise in $t_{m i x H}$ since mixing continuously reduces the gradients. Ultimately, at the start of ignition, gradients are again increased and $t_{\text {mixH }}$ reduce 


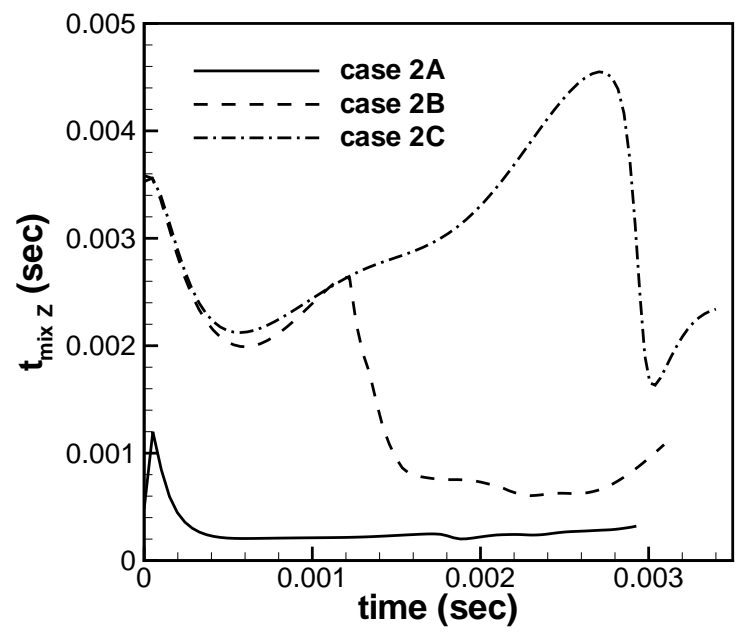

Figure 17. Average mixing time scale of mixture fraction as a function of time for cases (2A), (2B) and (2C).

sharply. The start of ignition creates sharp gradients in temperature and species mass fractions especially for Cases $(2 \mathrm{~A})$ and $(2 \mathrm{~B})$. Diffusion of temperature and species then increases the gradients of enthalpy across the reaction front, causing a reduction in $t_{\text {mixH }}$ at the onset of ignition. It is observed that turbulence reduces $t_{\text {mixH }}$ to values lower than ignition delay for all the three cases. Thus, passive mixing in enthalpy is important for all cases and causes the enthalpy fields to change significantly prior to ignition.

Passive mixing time scales in $Z$ is shown in Fig. 17 for all cases. Initial $t_{\text {mixz }}$ for Cases $(2 B)$ and $(2 C)$ is similar. $t_{\text {mix } Z}$ for Case $(2 \mathrm{~A})$ is undefined at the initial time since both $Z^{\prime}$ and $\widetilde{\chi_{Z}}$ are zero. It is again seen that turbulent straining increases gradients in $Z$ and causes the mixing time scales to reduce to values comparable to or lower than ignition delay for all cases. Subsequently, diffusion of species causes $Z^{\prime}$ and $\widetilde{\chi_{Z}}$ to become positive for Case $(2 \mathrm{~A})$, although $t_{\text {mix }}$ is still much smaller for Case $(2 \mathrm{~A})$ compared to Cases $(2 \mathrm{~B})$ and $(2 \mathrm{C})$. Further mixing reduces the gradients and an increase in mixing time scale is observed. The start of ignition increases the gradients and there is a sharp reduction in $t_{m i x z}$ for Cases $(2 B)$ and $(2 \mathrm{C})$ at the onset of ignition. For Case (2C) a large increase in $t_{m i x z}$ is seen prior to ignition, and it becomes greater than the corresponding ignition delay. This implies that passive mixing in $Z$ is not very important for Case $(2 \mathrm{C})$ after the initial drop in $t_{\text {mixz }}$. Passive mixing in $Z$ is, however, important for Cases (2A) and (2B) at all times.

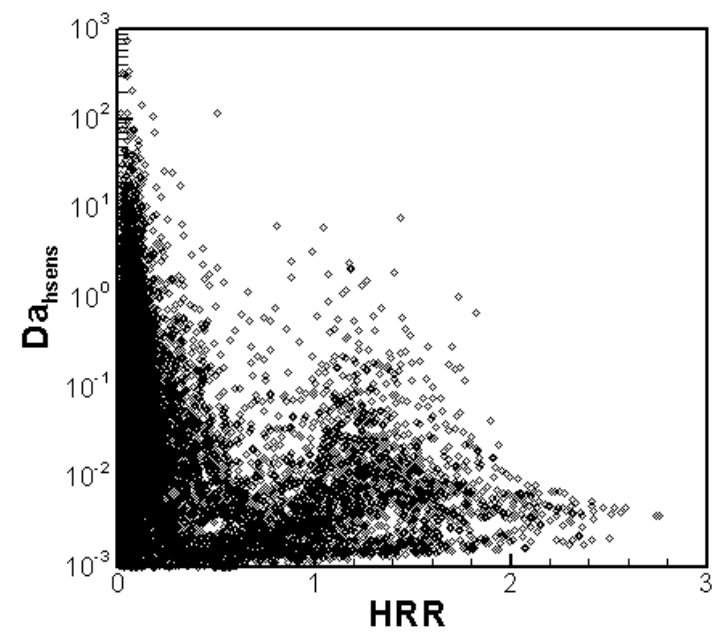

Figure 18. Scatter plot of $D a_{h s e n s}$ against HRR for Case (2A) at the $10 \%$ heat release point. 


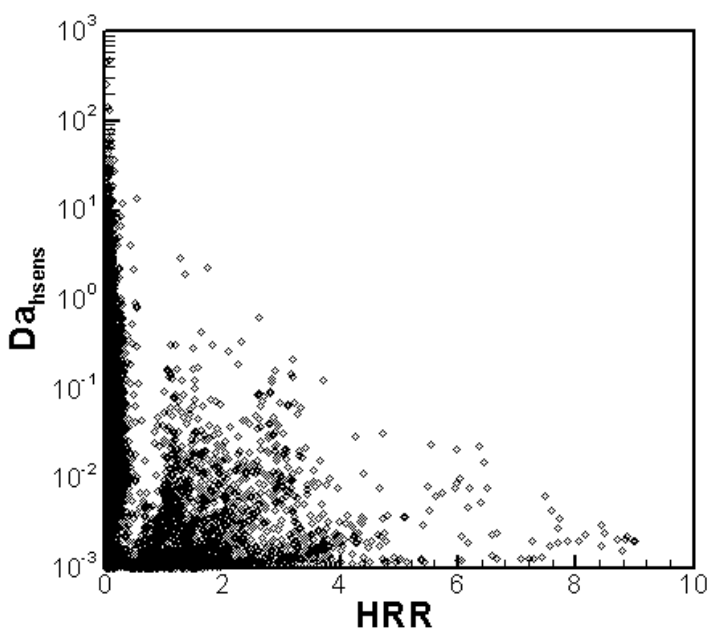

Figure 19. Scatter plot of Dahsens against HRR for Case (2B) at the $10 \%$ heat release point.

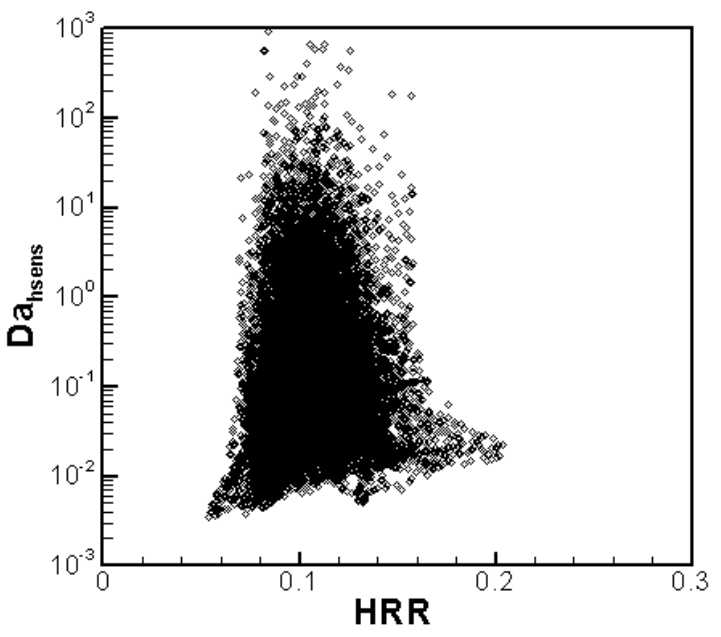

Figure 20. Scatter plot of Dahsens against HRR for Case (2C) at the $10 \%$ heat release point.

Next, the Damköhler number criterion developed in Section IV is applied to the 2D simulation results. Figures 18, 19 and 20 show the scatter plots of HRR against Dahsens at 10\% heat release point for Cases (2A), $(2 \mathrm{~B})$ and $(2 \mathrm{C})$, respectively. Figure 18 shows that majority of data points at high HRR values have Dahsens less than unity for Case (2A). This indicates that diffusion is dominant in most of high HRR locations, and thus deflagration front propagation is the dominant mode of ignition. A small number of high HRR locations do have Dahsens greater than one, and thus at those locations ignition occurs in the spontaneous ignition front propagation mode. For Case (2B), in Fig. 19 it can be seen that almost all locations of high HRR have Dahsens much smaller than unity. Therefore, ignition mode is predominantly in the deflagration front propagation in this case. Finally, Figure 20 shows distinct behavior. It is seen that the variation in HRR is much smaller for case (C) compared to other two cases. This shows that the entire region is reacting simultaneously, and large number of points have $\mathrm{Da}_{\text {hsens }}$ much greater than one. Thus, reaction is dominant compared to diffusion, and therefore, homogeneous ignition is the primary mode of heat release for this case. Cases (2B) and (2C) of 2D simulations qualitatively correspond to Cases (1B) and (1C) of 1D simulations, respectively.

Finally, we investigate the physical locations where spontaneous ignition front or premixed flames are formed. Figure 21 shows the temperature contours (in color) at $10 \%$ heat release point for the three cases. 


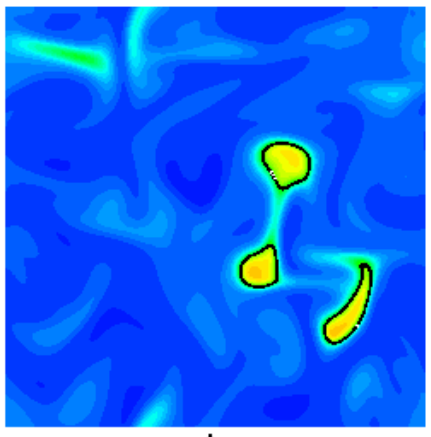

A

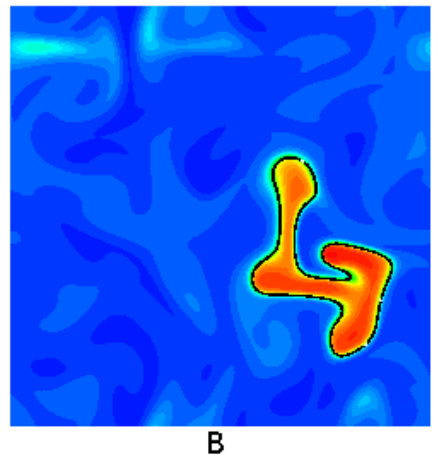

B

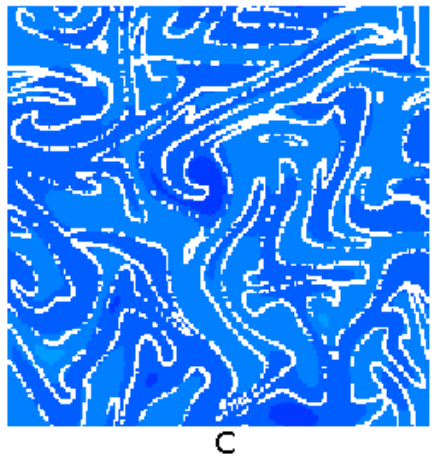

C

Figure 21. A: case (2A), B: case (2B), and C: case (2C). Temperature contours in color, levels ranging from 1050 K (blue) to $1600 \mathrm{~K}$ (red). Black contour in Cases (2A) and (2B) correspond to $Y_{\mathrm{H}_{2}}=7 \mathrm{e}-4$. White contours in all cases correspond to Dahsens $>0.5$

Black contour lines for Cases $(2 \mathrm{~A})$ and $(2 \mathrm{~B})$ shows $Y_{\mathrm{H}_{2}}=7 \mathrm{e}-4$. It is found that this $Y_{\mathrm{H}_{2}}$ iso-contour corresponds to locations of high HRR. Simultaneously, white iso-contours of Dahsens $>0.5$ are overlaid, which is taken as a nominal value where reaction and diffusion terms are comparable in magnitude. There are only a few white dots in Case (2A), which represents the locations where reaction is dominant, and almost no white spots in Case (2B), further demonstrating that the black iso-contour in Cases (2A) and (2B) is largely a premixed flame. Since the entire domain is found to be reacting in Case (2C) (see Fig. 20), the Damköhler number criterion is applied on the entire domain for this case. The widespread white iso-contours in Case $(2 \mathrm{C})$ again represent Dahsens $>0.5$, further confirm that Case $(2 \mathrm{C})$ is dominated by homogeneous ignition.

\section{Conclusions}

The autoignition and front propagation characteristics in LTC engine environments are studied computationally. In the first part of the study, non-reacting realistic 3D engine simulations were carried out to explore the different mixture formation scenarios existing in an LTC engine. Specifically, the influence of fuel spray injection timing on the correlation between temperature and equivalence ratio close to topdead center was investigated. It was observed that early fuel injection results in a largely uncorrelated temperature-equivalence ratio fields, whereas late fuel injection results in a largely negatively correlated temperature-equivalence ratio fields

Based the different mixture fields observed in the engine simulations, parametric studies of the various scalar mixing scenarios were conducted using high-fidelity DNS. First, one-dimensional simulations were performed for three different cases: Cases (1A) and (1B) with temperature fluctuations only at a different magnitude, and Case (1C) with a negatively correlated equivalence ratio fluctuation. It was found that for Cases (1A) and (1B) front propagation occurs after initial ignition kernel development. The consumption of end charge was much slower for Case (1B) with a larger level of temperature variation. Case (1C) represented a nearly homogeneous ignition. A Damköhler number based on sensible enthalpy (Dahsens) was defined to identify the roles of diffusion and reaction in the heat release process. In Case (1A), a large number of high heat release rate locations have Dahsens $>1$, suggesting spontaneous ignition front propagation. In Case (1B), Dahsens $<1$ for almost all high heat release rate locations implying diffusion to be dominant, and therefore ignition is dictated by deflagration front propagation. For Case (1C) Dahsens values much greater than one are observed for a large number of points, confirming that the mixture autoignites homogeneously without much diffusion effects.

Results of two-dimensional DNS were subsequently investigated for three cases: Case (2A) with only temperature fluctuations, Case $(2 \mathrm{~B})$ with uncorrelated temperature and equivalence ratio fields, and Case (2C) with negatively correlated temperature and equivalence ratio fields. Front propagation is observed for Cases (2A) and (2B) and homogeneous ignition is seen for Case (2C). Compared to Case (2A), ignition delay was found to decrease for Case (2B) and increase for Case (2C). Duration of heat release was increased for Case $(2 \mathrm{~B})$ and decreased for Case (2C) compared to Case (2A). Passive mixing time scale criterion 
developed in a previous study ${ }^{9}$ was applied to the three cases. Passive mixing in enthalpy scalar was found to be important for all cases and causes the enthalpy field to change significantly prior to ignition. On the other hand, passive mixing in mixture fraction scalar was found to be only important for Cases (2A) and $(2 \mathrm{~B})$ at all times, while for Case (2C) it loses significance after the initial drop in the mixing time scale $\left(t_{m i x z}\right)$. The Damköhler number criterion was found to be a useful computational diagnostic tool to identify the ignition modes in multi-dimensional ignition cases.

\section{Acknowledgments}

This study was supported by the University Consortium on Low Temperature Combustion for HighEfficiency, Ultra-Low Emission Engines directed by the University of Michigan and funded by the Department of Energy. The authors would like to thank Professor Matthias Ihme of University of Michigan for helpful comments during the course of the study.

\section{References}

${ }^{1}$ F. Zhao, T. Asmus, D. Assanis, J. Dec, J. Eng, P. Najt, Homogeneous Charge Compression Ignition (HCCI) Engines, Key Research and Development Issues, Society of Automotive Engineers, Inc., Warrendale, PA, 2002. pp. 1-14 and 531-541.

${ }^{2} \mathrm{~A}$ University consortium on low temperature combustion for high-efficienty ultra-low emission engines, directed by the University of Michigan, funded by Department of Energy under agreement DE-FC26-06NT42629.

${ }^{3}$ K. Epping, S. Aceves, R. Bechtold, J. Dec, SAE Technical Paper 2002-01-1923 (2002).

${ }^{4}$ M. Sjöberg, J. E. Dec, N. P. Cernansky, SAE Technical Paper 2005-01-0113 (2005).

${ }^{5}$ A. Babajimopoulos, G. A. Lavoie, D. Assanis, SAE Technical Paper 2003-01-3220 (2003)

${ }^{6}$ R. Hasegawa, H. Yanagihara, SAE Technical Paper 2003-01-0745 (2003).

${ }^{7}$ A. Hultqvist, M. Christenson, B. Johansson, M. Richter, J. Nygren, J. Hult, M. Alden, SAE Technical Paper 2002-01-0424 (2002).

${ }^{8}$ R. Sankaran, H. G. Im, E. R. Hawkes, J. H. Chen, Proc. Combust. Inst. 30 (2005) 875-882.

${ }^{9}$ J.H. Chen, E. R. Hawkes, R. Sankaran, S. D. Mason, H. G. Im, Combust. Flame 145 (2006) 128-144.

${ }^{10}$ E. R. Hawkes, R. Sankaran, P. P. Pébay, J. H. Chen, Combust. Flame 145 (2006) 145-159.

${ }^{11}$ M. A. Mueller, T. J. Kim, R. A. Yetter, F. L. Dryer, Int. J. Chem. Kinet. 31 (1999) 113-125.

${ }^{12}$ Y. B. Zel'dovich, Combust. Flame 39 (1980) 211-214.

${ }^{13}$ A. Babajimopoulos, D. Assanis, S. B. Fiveland, SAE Technical Paper 2002-01-2829 (2002)

${ }^{14}$ C. A. Kennedy, M. H. Carpenter, Appl. Num. Math. 14 (1994) 367458.

${ }^{15}$ C. A. Kennedy, M. H. Carpenter, R. M. Lewis, Appl. Num. Math. 35 (2000) 177264.

${ }^{16}$ M. D. Smooke, V. Giovangigli, in: M. D. Smooke (Ed.), Reduced Kinetic Mechanisms and Asymptotic Approximations for Methane-Air Flames, in: Lecture Notes in Physics, vol. 384, Springer-Verlag, New York (1991)

${ }^{17}$ R. J. Kee, F. M. Rupley, E. Meeks, J. A. Miller, Chemkin III: A Fortran Chemical Kinetics Package for the Analysis of Gas-Phase Chemical and Plasma Kinetics, Tech. Rep. SAND96-8216, Sandia National Laboratories, 1996

${ }^{18}$ J. O. Hinze, Turbulence, McGraw-Hill, New York (1975).

${ }^{19}$ C. Funk, V. Sick, D. L. Reuss, W. J. A. Dahm, SAE Technical Paper SAE 2002-01-2841 (2002).

${ }^{20}$ G. Bansal, H. G. Im, S. R. Lee, AIAA J. (2009) (in press)

${ }^{21}$ G. Bansal, H. G. Im, S. R. Lee, Proc. Combust. Inst., doi:10.1016/j.proci.2008.06.103 (2009)

${ }^{22}$ D. Bradley, C. Morley, X. J. Gu, D. R. Emerson, SAE Technical Paper 2002-01-2868 (2002).

${ }^{23}$ X. J. Gu, D. R. Emerson, D. Bradley, Combust. Flame 133 (2003) 63-74.

${ }^{24}$ R. Sankaran, H. G. Im, Combust. Theory Modelling 9 (2005) 417-432.

${ }^{25}$ R.W. Bilger, Proc. Combust. Inst. 22 (1988) 475-488. 